13

\title{
Глубокая рентгеновская рефлектометрия сверхмногопериодных A3B5-структур с квантовыми ямами, выращиваемых методом молекулярно-пучковой эпитаксии
}

\author{
(С) Л.И. Горай, ${ }^{1,2,3}$ Е.В. Пирогов, ${ }^{1,4}$ М.С. Соболев, ${ }^{1}$ Н.К. Поляков, ${ }^{1}$ А.С. Дашков, ${ }^{1}$ М.В. Свечников, ${ }^{5}$ \\ А.Д. Буравлев ${ }^{1,6}$
}

${ }^{1}$ Академический университет им. Ж.И. Алферова, 194021 Санкт-Петербург, Россия

2 Университет ИТМО, 197101 Санкт-Петербург, Россия

${ }^{3}$ Институт аналитического приборостроения РАН, 190103 Санкт-Петербург, Россия

${ }^{4}$ ООО „Коннектор Оптикс“, 194292 Санкт-Петербург, Россия

${ }^{5}$ Институт фризики микроструктур РАН, 607680 Нижний Новгород, Россия

${ }^{6}$ Физико-технический институт им. А.Ф. Иофффе РАН, 194021 Санкт-Петербург, Россия

e-mail: lig@pcgrate.com

Поступило в Редакцию 2 апреля 2020 г.

В окончательной редакции 2 апреля 2020 г.

Принято к публикации 2 апреля 2020 г.

Нами исследовались упруго-напряженные $\mathrm{AlGaAs} / \mathrm{GaAs}$ сверхмногопериодные (СМП - 100-1000 периодов) сверхрешетки с различной степенью легирования и небольшой разницей в толщине периода. Предложенная методика характеризации, состоящая из согласованного применения метода глубокой рентгеновской рефлектометрии, основанного на строгом методе расчета, а также известного метода высокоразрешающей рентгеновской рефлектометрии, позволили исследовать 100-периодные структуры с 2-nm ширинами $\mathrm{Al}_{0.3} \mathrm{Ga}_{0.7} \mathrm{As}$ барьеров и 10 -nm ширинами $\mathrm{GaAs}$ ям и с высокой точностью определить толщины слоев и размытость интерфейсов, что можно рассматривать как первый шаг на пути дальнейшего анализа толстых структур на ярких источниках синхротронного излучения. Разница ожидаемых и получившихся в результате восстановления предложенным методом значений толщин слоев составила несколько процентов, в том числе для образцов с высокой степенью легирования (до $10^{18} \mathrm{~cm}^{-3}$ ). Все СМП структуры характеризуются резкими интерфейсами со среднеквадратичным отклонением порядка $0.1 \mathrm{~nm}$. На основе полученных данных толщин можно точно определять состав слоев с помощью высокоразрешающей рентгеновской дифрактометрии.

Ключевые слова: сверхрешетка, AlGaAs-гетероструктура, рентгеновская рефлектометрия, строгая электромагнитная теория рассеяния.

DOI: 10.21883/JTF.2020.11.49982.108-20

\section{Введение}

В последнее время все большую актуальность приобретает создание терагерцовых источников излучения, в том числе перестраиваемых источников, которые могут работать при комнатных температурах. Подобные полупроводниковые излучающие структуры могут быть использованы как для медицинских диагностических систем, так и систем безопасности $[1,2]$. Однако, в настоящий момент перестраиваемых источников на данный диапазон частот фактически не существует. Нами разрабатываются перспективные А3B5 структуры с большим числом периодов (от 100 до 1000) и толщиной до $12 \mu \mathrm{m}$, выращиваемые на установке полупромышленного типа Riber 49 методом молекулярно-пучковой эпитаксии (МПЭ) и обладающие резкими гетерограни- цами, постоянством состава и хорошей поверхностной однородностью [3,4]. Для синтеза подобных образцов необходимо создать новые методы прецизионного контроля структурных параметров и состава, в первую очередь, толщин периодов и ширин интерфейсов, в том числе с использованием строгих теоретических методов и данных, получаемых на источниках синхротронного излучения (СИ) с ультрамалым эмиттансом. В настоящей работе рентгеновскими методами исследовались упруго-напряженные $\mathrm{AlGaAs} / \mathrm{GaAs}$ сверхмногопериодные (СМП) сверхрешетки (СР) с различной степенью легирования и малой разницей в толщинах слоев. Предложенная лабораторная методика характеризации, состоящая из согласованного применения нового теоретического метода глубокой рентгеновской рефлектометрии (ГРР) и известного метода высокоразрешаю- 
щей рентгеновской рефлектометрии (ВРP), позволяет исследовать 100-периодные структуры с 2-nm ширинами $\mathrm{Al}_{0.3} \mathrm{Ga}_{0.7} \mathrm{As}$ барьеров в и 10-nm ширинами $\mathrm{GaAs}$ ям на лабораторных источниках, и с высокой точностью определить толщины слоев и шероховатость/диффузность границ, что можно рассматривать как первый шаг на пути анализа толстых структур на ярких источниках СИ. Метод ГРР уже успешно применялся для анализа многослойных кристаллических и аморфных образцов большой толщины, предназначенных для создания приборов микроэлектроники и рентгеновской оптики [5-8]. Данный подход основан на строгом методе теории дифракции электромагнитного излучения [9], который работает от полного внешнего отражения до полного поглощения коротковолнового излучения с возможностью точного определения интенсивности рассеянного света и поглощения, что позволяет проверять приближенные методы и анализировать СМП структуры с помощью измерений на источниках СИ и рентгеновских лазерах на свободных электронах. Разница ожидаемых и получившихся в результате восстановления методом ГРР и ВРР значений толщин слоев должна составлять не более нескольких процентов, в том числе для образцов с высокой степенью легирования (до $10^{18} \mathrm{~cm}^{-3}$ ). Подобные структуры должны характеризоваться резкими интерфейсами со среднеквадратичным отклонением (СКО) ширины переходных слоев порядка нескольких $0.1 \mathrm{~nm}$. Опираясь на полученные с помощью разработанной методики согласованные данные толщин слоев, с помощью метода высокоразрешающей рентгеновской дифрактометрии (ВРД) можно определить состав слоев СР. Методике согласованного применения методов ВРР и ВРД для анализа данного типа СМП структур будет посвящена отдельная публикация.

\section{1. Теория}

В строгом смысле факторы Дебая-Валлера (DW) и Нево-Кросе (NC), широко используемые для учета уменьшения зеркального отражения, действительны только в случае малой высоты случайных неровностей границы $h$ (или СКО шероховатости $\sigma$ ), имеющих гауссовское распределение и очень большую (DW) или очень малую (NC) длину корреляции $\xi$. Борновское приближение деформированных волн второго порядка (DWBA) может использоваться для учета произвольных величин $\zeta$, однако, в общем случае оно справедливо для малых значений $2 \pi \sigma \sin \theta / \lambda$ и при использовании гауссово-подобной корреляционной функции, где $\theta-$ угол скольжения, отсчитываемый от поверхности, $\lambda-$ длина волны $[10,11]$. Кроме того, при выводе фактора $\mathrm{NC}$ предполагается, что $\xi$ удовлетворяет $\lambda \gg 2 \pi \xi \sin ^{2} \theta$. Теория возмущения по высоте шероховатостей является более общей в смысле использования любого статистического распределения неровностей по высоте и любой корреляционной функции, однако она также имеет силь- ные ограничения, главным образом, на максимальные значения $h[12,13]$. Большая разница (до порядка и более величины) в зеркальных коэффициентах отражения между строгим подходом и DW или NC аппроксимациями была впервые теоретически продемонстрирована на примере Аu-зеркал с различными параметрами шероховатости и длинами волн, падение излучения на которые происходит при углах скольжения, близких или больше критического [14]. Эта разница может привести к неправильным оценкам $\sigma$ и $\xi$, если они получены из ВРР путем сравнения экспериментальных данных с расчетными [15]. С помощью аналогичного строгого подхода были рассчитаны коэффициенты зеркального и диффузного отражения для многослойных $\mathrm{Al} / \mathrm{Zr}$ зеркал с учетом реальной (негауссовой) статистики шероховатых границ в экстремальном ультрафиолетовом диапазоне длин волн [16]. Сложная многослойная модель [17], точно учитывающая эффекты кинетики роста структурированных границ, имеющих случайные шероховатости с различными углами наклона граней и величинами $\sigma$ и $\zeta$, демонстрирует хорошую корреляцию значений зеркальной отражательной способности с экспериментальными данными, полученными на источнике СИ для различных углов падения и длин волн.

Чтобы строго рассчитать интенсивность рассеяния на шероховатых поверхностях с использованием электромагнитного кода PCGrate ${ }^{\mathrm{TM}}$-SX v. 6.7 [18], мы применяем метод Монте Карло для усреднения статистики шероховатости по ансамблю реализаций отдельных поверхностей. В данном случае можно использовать модель вертикально некоррелированных шероховатостей из-за значительного случайного шума источников материала и малого $\sigma$ (около нескольких $0.1 \mathrm{~nm}$ ) подложек [16,19]. В этом случае зеркальное отражение СР пропорционально зеркальному отражению на границах. Более того, простая оценка показывает, что угловое распределение интенсивности рассеяния аналогично рассеянию на одной границе [20]. Таким образом, мы можем детально изучить с помощью строгого метода только одну шероховатую поверхность GaAs, что гораздо легче, и сравнить результаты расчетов с использованием ГРР и факторов ослабления DW или NC, которые применяются для решения обратных задач ВРP.

С помощью использования приближенных теоретических подходов на основе поправок DW и NC на рис. 1 показано, какое влияние оказывает величина $\sigma$ на отток интенсивности зеркального ТЕ отражения (данные коэффициента отражения для ТМ поляризации близки по величине для всех приведенных кривых) поверхности $\mathrm{GaAs}$, в зависимости от $\theta$ и $\lambda=0.1541 \mathrm{~nm}$. Из рисунка следует, что вблизи критического угла коэффициенты зеркального отражения для обеих моделей с шероховатостью $\sigma=0.35 \mathrm{~nm} \mathrm{c} \mathrm{высокой} \mathrm{точностью} \mathrm{близки}$ друг к другу и коэффициенту отражения идеальногладкой поверхности. Для модели NC с $\sigma=0.7$ и, даже, $\sigma=1.5 \mathrm{~nm}$ результаты мало (не более нескольких \%) отличаются от идеального отражения. Для модели DW и 


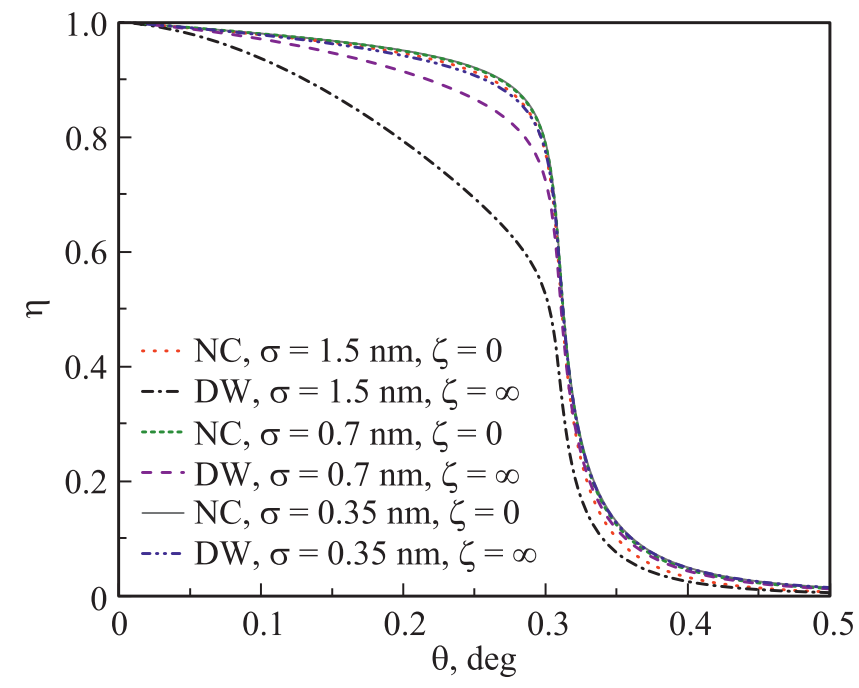

Рис. 1. Коэффициент зеркального отражения $\eta \mathrm{GaAs}$, pacсчитанный приближенно с помощью поправки NC или DW для длины волны $\lambda=0.15406 \mathrm{~nm}$, в зависимости от угла скольжения $\theta$ вблизи критического угла.

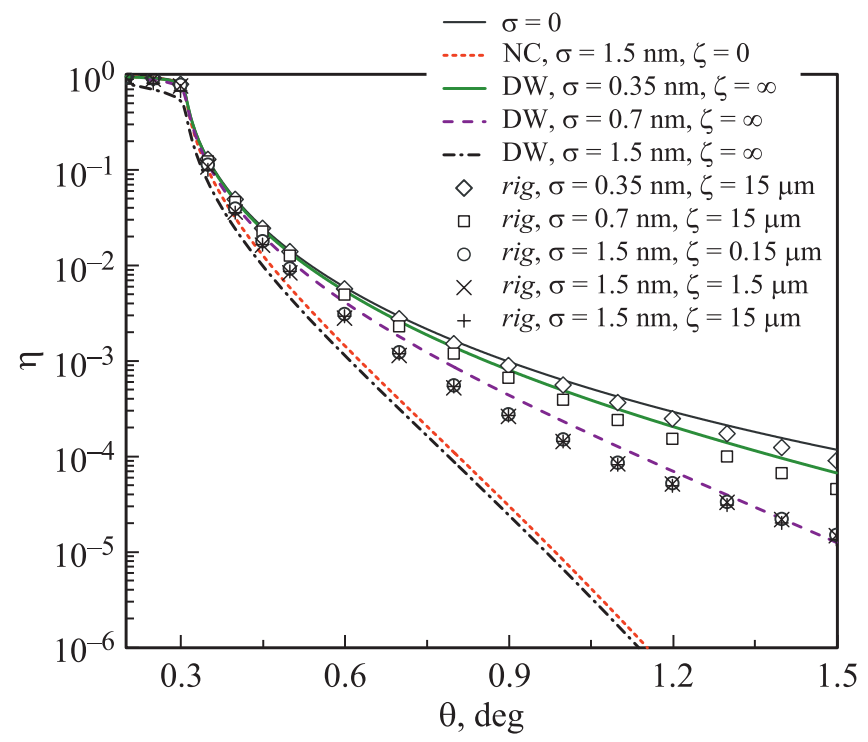

Рис. 2. Коэффициент зеркального отражения $\eta \mathrm{GaAs}$, pacсчитанный приближенно с помощью поправки NC или DW (кривые) и строго (rig - символы) с 99 статистическими реализациями поверхности для длины волны $\lambda=0.1541 \mathrm{~nm}$ и различной среднеквадратичной шероховатостью $\sigma$ и длиной корреляции $\xi$, в зависимости от угла скольжения $\theta$ в широком диапазоне углов.

$\sigma=0.7$ или $\sigma=1.5 \mathrm{~nm}$ результаты уже значительно (от нескольких \% до нескольких десятков \%) отличаются от идеального отражения вблизи критического угла. С другой стороны, на рис. 2 хорошо видна разница между результатами, полученными с использованием приближенных подходов и строгих расчетов, которые выполнены для различных значений $\sigma$ и $\zeta$. Для борновского приближения (фактор DW) $\xi=\infty$, для DWBA первого порядка (коэффициент NC или более общее приближение Синха [21]) $\xi=0$, а для выбранной строгой модели величины $\xi$ имеют промежуточные реалистичные значения. Сравнение коэффициентов отражения для $\lambda=0.1541 \mathrm{~nm}$, полученных для приближенной (DW или $\mathrm{NC})$ и совершенной $(\sigma=0)$ моделей и строгой модели для различных $\sigma$ и $\xi$, показано на рисунке в логарифмическом масштабе в широком угловом диапазоне. Для $\sigma=1.5 \mathrm{~nm}$ и $\xi=0.15 \mu \mathrm{m}$ результаты, полученные для строгой модели и модели $\mathrm{NC}$, отличаются лишь на $1-2 \%$ вблизи и ниже критического угла и примерно на несколько процентов в диапазоне, немного превышающем критический угол. Для $\xi=1.5 \mu \mathrm{m}$ эта разница составляет около нескольких \% в диапазоне высокого коэффициента отражения и около нескольких десятков \% в диапазоне углов, несколько больших критического. Для $\xi=15 \mu \mathrm{m}$ разница составляет уже один-два десятка \% в диапазоне высоких коэффициентов отражения и около нескольких десятков \% или, даже, несколько раз в диапазоне углов, несколько больше критического. Подобные различия, около нескольких десятков \% в диапазоне высоких коэффициентов отражения и около нескольких десятков \% или нескольких раз в диапазоне низких коэффициентов отражения, можно видеть на рис. 2 для $\xi=15 \mu \mathrm{m}$ и модели DW. Для $\sigma=0.7 \mathrm{~nm}$ модель $\mathrm{NC}$ (не показана на рис. 2) близка, с максимальной разницей в несколько \%, к идеальному коэффициенту отражения, а также к строгой модели с наибольшим приведенным значением $\xi=15 \mu \mathrm{m}$. Для $\sigma=0.35 \mathrm{~nm}$ все модели очень близки (с максимальной разницей в несколько \%) в рассмотренном диапазоне углов падения.

Для диапазона угла падения, намного превышающего критический угол, результаты, полученные для строгой модели и моделей DW и $\mathrm{NC}$, отличаются значительно больше. Для $\sigma=1.5 \mathrm{~nm}$ на рис. 2 строгие результаты, полученные для различных $\xi$, близки, однако они очень далеки от приближений DW и NC. Например, для $\theta=0.9^{\circ}$ результаты различаются на порядок, а для больших значений $\theta-$ до нескольких порядков. Для $\sigma=0.7 \mathrm{~nm}$ и $\xi=15 \mu \mathrm{m}$ строгие результаты наиболее близки к предсказаниям модели DW, однако разница варьируется от нескольких десятков \% до нескольких раз в исследуемом диапазоне углов. Для $\sigma=0.35 \mathrm{~nm}$ и $\xi=15 \mu \mathrm{m}$ на рис. 2 строгие результаты близки к предсказаниям модели DW, причем разница в исследуемом диапазоне варьируется от нескольких \% до нескольких десятков \%. Представленные результаты строгих расчетов демонстрируют хорошую сходимость и высокую точность, необходимые для моделирования зеркального отражения СР с полигональными случайношероховатыми границами, имеющими 1000 узлов и исследуемыми в широком угловом диапазоне падения излучения. B PCGrate мы использовали 1000-1200 точек дискретизации на границу, 99-195 реализаций случайных границ и „Разделяющий“ („Separating“) солвер с выключенной опцией ускорения сходимости; и получили ошибку $\sim 10^{-6}$, оцененную из энергетического 
баланса с учетом точного вычисления поглощения [9]. Разница между коэффициентами отражения, полученными с использованием 99 и 195 наборов случайных границ, составляет $\sim 0.1 \%$. Разница между коэффициентами отражения, полученными с использованием 49 и 195 наборов случайных границ, составляет не более $\sim 10 \%$. Среднее время, затрачиваемое на одну расчетную точку (99 случайных реализаций) на портативной рабочей станции MSIß WT73VR 7RM с процессором Intel $($ Xeon $($ E3-1505M V6 \& 3-4 GHz и $64 \mathrm{~GB}$ O3У, составляет $\sim 4 \mathrm{~h}$ при работе под Windows $\AA 10$ Pro и использовании восьмикратного распараллеливания. Показатели преломления GaAs и AlGaAs были получены с помощью данных веб-сайта CXRO [22].

Таким образом, как и предсказывает общая теория, строгие результаты для малых значений $\sigma$ и небольших значений $\xi$ близки к модели NC вблизи и немного ниже критического угла. При других значениях исследуемых параметров, точные результаты могут быть близки к модели DW или NC в диапазоне выше критического угла, но только для небольших значений $\sigma$ - около или менее $0.35 \mathrm{~nm}$ для рассматриваемых структур и материалов. При более высоких значениях $\sigma$ такие явные различия между строгой моделью и любыми широко используемыми приближениями могут привести к переоценке $\sigma$, если она получена из сравнения экспериментальных данных с расчетами, что имеет место, например, для аналогичных толстых структур Туре I, рассмотренных в [3].

\section{2. Эксперимент}

В настоящей работе исследовались несколько видов образцов, состоящих из 100 периодов слоев $\mathrm{Al}_{0.3} \mathrm{Ga}_{0.7} \mathrm{As}$ и GaAs толщиной $2 \mathrm{~nm}$ и $10 \mathrm{~nm}$ соответственно, и выращенных на „ері-ready“ подложках GaAs с ориентацией (100) методом МПЭ в полупромышленной установке Riber 49. Качество слоев контролировалось in situ системой дифракции быстрых электронов на отражение. Данный тип структур имел толщину $\sim 1.2 \mu \mathrm{m}$ без учета толщины подложки. Структуры значительно отличались концентрацией легирующей примеси, от нелегированных до $n \approx 10^{18} \mathrm{~cm}^{-3}$, и незначительно - толщинами слоев. Кремний использовался в качестве донорной легирующей примеси $n$-типа. Уровни легирования по всей толщине СР были примерно одинаковыми для барьеров $\mathrm{Al}_{0.3} \mathrm{Ga}_{0.7} \mathrm{As}$ и ям $\mathrm{GaAs}$ и составляли от $n \approx 10^{16}$ до $10^{18} \mathrm{~cm}^{-3}$.

Полученные структуры исследовались методами ВРР и ВРД. Рефлектометрические измерения были произведены на дифрактометре PANalytical X'PertPro c характеристической длиной волны излучения $\mathrm{Cu} K_{\alpha 1}$ $(\lambda=0.15406 \mathrm{~nm})$ при скользящих углах падения в диапазоне $\Delta \theta=0-4^{\circ}$ с шагом сканирования $0.0005^{\circ}$ и щелью $\omega=1 \mathrm{~mm}$. Дифрактометрическая съемка структур была произведена в режиме $\theta-2 \theta$ сканирования в параллельной геометрии пучка. Использовалась схема с рентгеновской трубкой мощностью $3 \mathrm{~kW}$ с характеристической длиной волны излучения $\mathrm{Cu} K_{\alpha 1}$ и четырехкратным Ge (220) прорезным кристалл-монохроматором с $\omega=1 \mathrm{~mm}$.

\section{3. Результаты и обсуждение}

На рис. 3 показаны рентгеновские дифракционные кривые вблизи симметричного (002) рефлекса GaAs. Моделирование полученных данных с использованием встроенных программных пакетов показало небольшое отклонение толщины периода СР от начальных значений. Значения периода составили $D=12.69 \pm 0.04$, $13.03 \pm 0.06$ и $12.84 \pm 0.07 \mathrm{~nm}$, соответственно, для нелегированной структуры, с легированием $n \approx 10^{17}$ и $10^{18} \mathrm{~cm}^{-3}$. Измерение нелегированной структуры производилось с использованием кристалла-анализатора, поэтому наблюдается отличие интенсивности сателлитных пиков от двух других кривых. Однако, это не препятствует точному определению значений периода, толщин слоев и ширин интерфейсов.

Полученная модель ВРР дает хорошее совпадение экспериментальных и теоретических пиков для восьми порядков дифракции, что говорит о высоком качестве структуры и хорошей точности подгонки (рис. 4-6). Параметры толщин слоев и интерфейсов структур, полученные в результате исследования методом ВРР, приведены в таблице. Значения периодов для двух методов (ВРР и ВРД) хорошо коррелируют. Следует отметить, что данные, полученные из анализа толщинных осцилляций Киссига в ВРР, являются более точными и используются нами для корректировки модели ВРД, так как они анализируются не по ширине пика, а

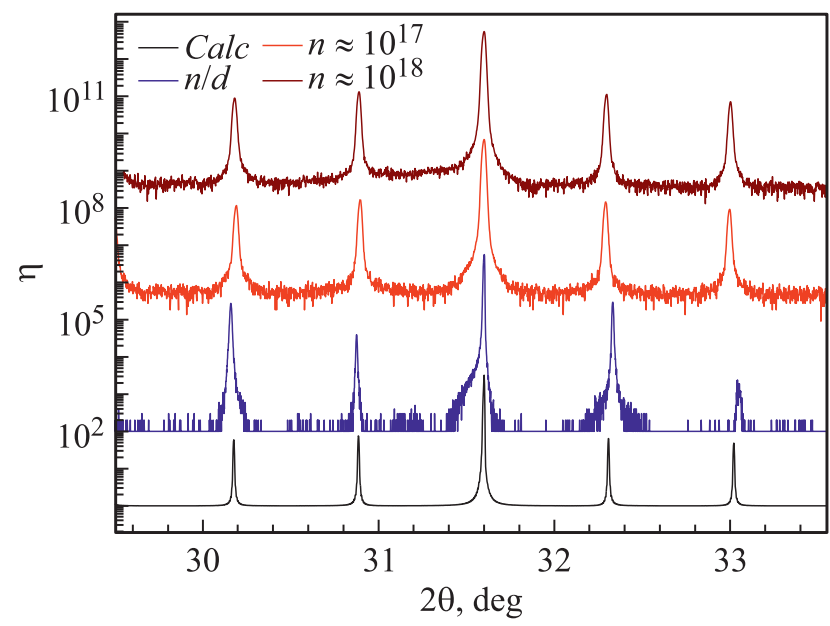

Рис. 3. Интенсивность дифракции $\eta$ структуры со 100 периодами и различным уровнем легирования $n$, полученная в режиме сканирования $\theta-2 \theta$ на длине волны $\lambda=0.15406 \mathrm{~nm}$ вблизи отражения (002) GaAs (кривые сдвинуты для наглядности). Для сравнения приведены расчеты (calc) для структуры $\mathrm{Al}_{0.3} \mathrm{Ga}_{0.7} \mathrm{As}(2.15 \mathrm{~nm}) / \mathrm{GaAs}(10.75 \mathrm{~nm})$. 
Восстановленные толщины слоев СМП структур с различным уровнем легирования

\begin{tabular}{|c|c|c|c|c|c|c|}
\hline \multirow{2}{*}{ Слой СР } & \multicolumn{2}{|c|}{ Нелегированный } & \multicolumn{2}{|c|}{$n \sim 1 \cdot 10^{17} \mathrm{~cm}^{-3}$} & \multicolumn{2}{|c|}{$n \sim 1 \cdot 10^{18} \mathrm{~cm}^{-3}$} \\
\hline & $\mathrm{BPP}, \mathrm{nm}$ & $\sigma, \mathrm{nm}$ & $\mathrm{BPP}, \mathrm{nm}$ & $\sigma, \mathrm{nm}$ & $\mathrm{BPP}, \mathrm{nm}$ & $\sigma, \mathrm{nm}$ \\
\hline GaAs & 10.60 & 0.39 & 10.89 & 0.34 & 10.78 & 0.41 \\
\hline AlGaAs & 2.11 & 0.42 & 2.13 & 0.43 & 2.11 & 0.46 \\
\hline$D$ & $12.71 \pm 0.02$ & & $13.02 \pm 0.04$ & & $12.89 \pm 0.06$ & \\
\hline
\end{tabular}

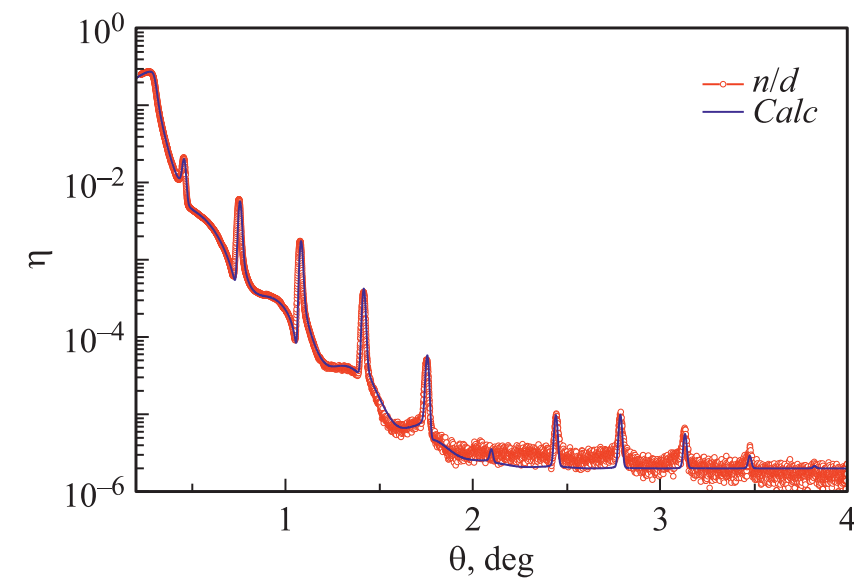

Рис. 4. Коэффициент зеркального отражения $\eta$ нелегированной $(n / d)$ структуры со 100 периодами, полученный для длины волны $\lambda=0.1541 \mathrm{~nm}$, в зависимости от угла скольжения $\theta$ (расчеты - calc).

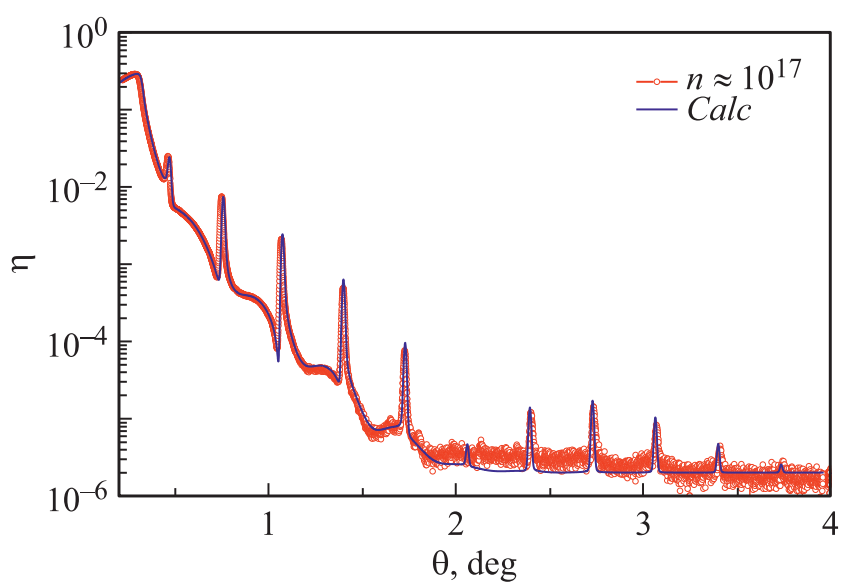

Рис. 5. Коэффициент зеркального отражения $\eta$ структуры со 100 периодами и уровнем легирования $n \sim 1 \cdot 10^{17} \mathrm{~cm}^{-3}$, полученный для длины волны $\lambda=0.1541 \mathrm{~nm}$, в зависимости от угла скольжения $\theta$ (расчеты - calc).

по его положению и усредняются по наблюдаемым осцилляциям в нескольких брэгговских порядках. Таким образом, периоды выращенных структур отличались от задаваемых при росте величин на 5-8\%, как и полученные значения толщин слоев, что может быть ском- пенсировано в программе роста с учетом полученных знаний. Наблюдаемые ширины дифракционных пиков и восстановленные значения $\sigma$ для всех образцов примерно одинаковы, что говорит о незначительном влиянии концентрации легирующей примеси до $n \approx 10^{18} \mathrm{~cm}^{-3}$ на качество получаемых структур.

Что касается исследуемых в настоящей работе СМП структур, теоретические результаты, полученные с помощью ГРР, показывают, что точные результаты могут быть близки к модели DW или NC (рис. 2), используемой при восстановлении параметров структур в широком диапазоне углов ВРР, но только для небольших значений $\sigma$ - около или менее $0.35 \mathrm{~nm}$ для рассматриваемых структур и материалов и практически любых корреляционных длин шероховатости. Таким образом, сравнение результатов решения прямой задачи рассеяния, полученных нами с помощью приближенных и строгого метода (ГРР), позволило не только оценить критическое значение $\sigma$, при котором расхождение данных интенсивности рассеяния становится значимым, но и определить величину конкретного расхождения зеркальных коэффициентов отражения путем нахождения точного решения прямой задачи в нескольких точках интересующего диапазона параметров. С учетом этого восстановленные с помощью ВРР величины $\sigma$ для слоев СР (см. таблицу) дают ошибку всего несколько \%

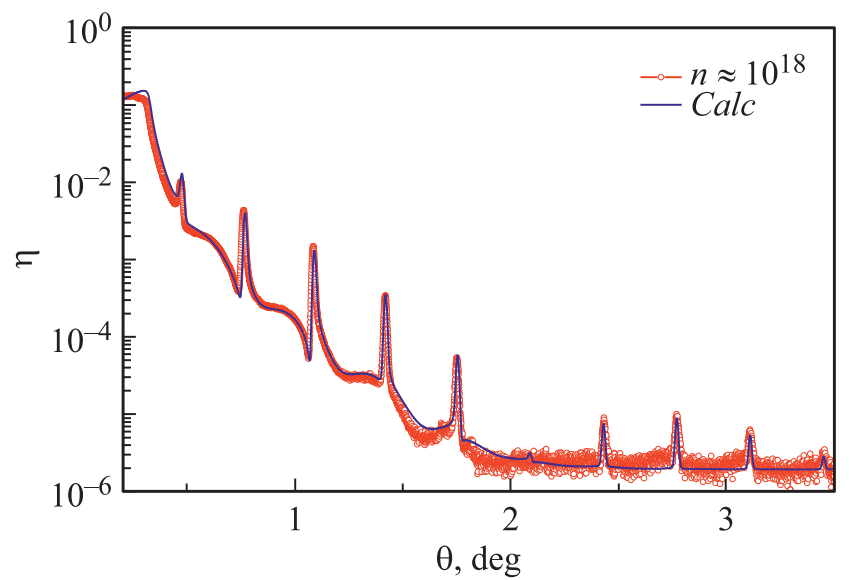

Рис. 6. Коэффициент зеркального отражения $\eta$ структуры со 100 периодами и уровнем легирования $n \sim 1 \cdot 10^{18} \mathrm{~cm}^{-3}$, полученный для длины волны $\lambda=0.1541 \mathrm{~nm}$, в зависимости от угла скольжения $\theta$ (расчеты - calc). 


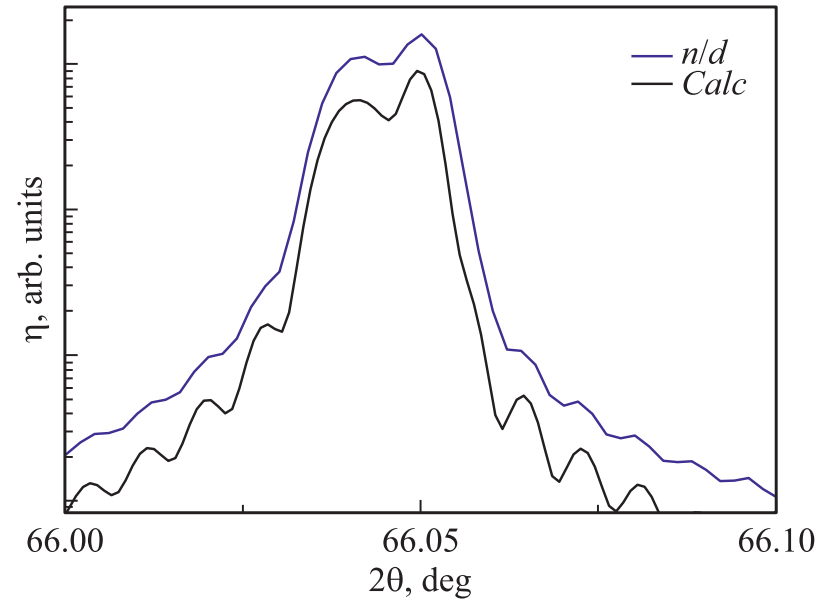

Рис. 7. Интенсивность дифракции $\eta$ (arb. units), полученная для нелегированной структуры в режиме сканирования $\theta-2 \theta$ в малом диапазоне углов на длине волны $\lambda=0.15406 \mathrm{~nm}$ вблизи отражения (004) GaAs (расчеты - calc).

в широком диапазоне исследованных углов падения, по сравнению с истинными значениями шероховатости. Следовательно, в данном случае используемая модель ВРР применима, и ошибка восстановления параметра $\sigma$ не хуже, чем общая ошибка восстановления параметров на основе ВРР.

Используя полученные значения толщин и положение пика от среднего состава для нелегированной структуры (рис. 7), была определена молярная доля $\mathrm{Al}$ в твердом растворе $\mathrm{Al}_{x} \mathrm{Ga}_{1-x} \mathrm{As}$, которая составила $x=0.30 \pm 0.005$.

\section{Заключение}

Рассмотренная методика исследований с помощью ГРР и ВРР, а также ВРД хорошо подходит для анализа СМП структур и позволяет охарактеризовать образцы и определить с высокой достоверностью и точностью такие параметры, как толщина слоев и шероховатость интерфейсов. Различия между ожидаемыми и полученными данными толщин слоев составляют несколько процентов для образцов рассмотренного типа с различной степенью легирования. Эти различия могут быть обусловлены недостаточно высокой точностью начальных калибровок, а также изменением потоков материалов 3 группы во время роста толстых структур, что может быть компенсировано в процессе проведения трудоемкого и дорогостоящего технологического эксперимента. Остаточное количество материала, а также его однородность в камере также влияют на изменение толщины слоя и ширину переходных областей. Согласно данным ГРР и ВРР, все структуры, в том числе с высоким уровнем легирования, характеризуются четкими границами с $\sigma$ порядка нескольких $0.1 \mathrm{~nm}$. Наши исследования показали, что метод ГРР, основанный на строгой модели рефлектометрии, необходимо использовать для проверки достоверности результатов ВРР, которые могут отличаться для восстанавливаемых коэффициентов зеркального отражения на десятки \% вблизи критического угла и на порядки величины - при больших углах скольжения. Для исследованных образцов и диапазонов углов величина $\sigma \sim 0.35 \mathrm{~nm}$ оказалась критичной для использования приближенных поправок типа DW и $\mathrm{NC}$, однако, в общем случае, это зависит от корреляционной длины (статистики шероховатости), угла падения и длины волны излучения и может быть определено только на основе применения строгой теории рассеяния. Опираясь на полученные согласованные данные, можно найти лучшее решение обратных задачи ВРР и ВРД [3] и определить изменения толщин и состава слоев СМП структур с высокой точностью.

Стоит также отметить, что аналогичные СМП структуры с числом периодов 300-400 и, даже, 1000 были выращены с использованием метода МПЭ и их исследования на основе разработанных нами методов, а также с применением источников СИ находятся в процессе. Таким образом, работа рассматривается как первый основной шаг в получении и рефлектометрическом анализе необходимых СМП гетероструктур с большим числом периодов и высоким уровнем легирования.

\section{Финансирование работы}

Работа частично поддержана Министерством образования и науки Российской Федерации (Минобрнаука) (FSRM-2020-0008) и Российским фондом фундаментальных исследований (РФФИ) (19-29-12053) в части экспериментальных исследований. Работа Л.И. Горая, Е.В. Пирогова, М.С. Соболева и А.С. Дашкова поддержана Российским научным фондом (РНФ) (19-12-00270) в теоретической части.

\section{Конфликт интересов}

Авторы заявляют, что у них нет конфликта интересов.

\section{Список литературы}

[1] Gmachl C., Sivco D.L., Colombelli R., Capasso F., Cho A.Y. // Nature. 2002. Vol. 415. P. 883-887. DOI: $10.1038 / 415883 \mathrm{a}$

[2] Андронов А.А., Додин Е.П., Зинченко Д.И., Ноздрин Ю.Н., Ладугин М.А., Мармалюк А.А., Падалища А.А., Беляков В.А., Ладенков И.В., Фефелов А.Г. // Письма в ЖЭТФ. 2015. Т. 102. Вып. 4. С. 235-239. [Andronov A.A., Dodin E.P., Zinchenko D.I., Nozdrin, Yu.N., Ladugin M.A., Marmalyuk A.A., Padalitsa A.A., Belyakov V.A., Ladenkov I.V., Fefelov A.G. // JETP Lett. 2015. Vol. 102. P. 207-211.] DOI: $10.7868 / \mathrm{S} 0370274 X 15160031$

[3] Goray L.I., Pirogov E.V., Sobolev M.S., Ilkiv I.V., Dashkov A.S., Vainer Yu.A., Svechnikov M.V., Yunin P.A., Chkhalo N.I., Bouravlev A.D. // Semiconductors. 2019. Vol. 53. N 14. P. $1910-1913$. DOI: $10.1134 / \mathrm{S} 1063782619140082$ 
[4] Goray L.I., Pirogov E.V., Nikitina E.V., Ubyivovk E.V., Gerchikov L.G., Ipatov A.N., Dashkov A.S., Sobolev M.S., Ilkiv I.V., Bouravlev A.D. // Semiconductors. 2019. Vol. 53. N 14. P. 1914-1917. DOI: 10.1134/S1063782619140094

[5] Goray L.I. // Proc. SPIE. 2007. Vol. 6617. P. 661719. DOI: $10.1117 / 12.726038$

[6] Горай Л.И., Чхало Н.И., Цырлин Г.Э. // ЖТФ. 2009. T. 79. Вып. 4. С. 117-124. [Goray L.I., Chkhalo N.I., Tsyrlin G.E. // Tech. Phys. 2009. Vol. 54. N 4. P. 561-568. DOI: $10.1134 / \mathrm{S} 1063784209040185]$

[7] Горай Л.И., Чхало Н.И., Вайнер Ю.А. // Письма в ЖТФ. 2010. Т. 36. Вып. 3. С. 31-38. [Goray L.I., Chkhalo N.I., Vainer Yu.A. // Tech. Phys. Let. 2010. Vol. 36. N 2. P. 108-111. DOI: 10.1134/S1063785010020057]

[8] Goray L., Lubov M. // Opt. Express. 2015. Vol. 23. N 8. P. 10703-10713. DOI: 10.1364/OE.23.010703

[9] Goray L.I., Schmidt G. Boundary Integral Equation Methods for Conical Diffraction and Short Waves, in Gratings: Theory and Numerical Applications / ed. E. Popov. 2nd rev. ed., Institut Fresnel, AMU, 2014. P. 447-536. URL: https://www.fresnel.fr/files/gratings/Second-Edition/index.htm.

[10] Goray L., Lubov M. // J. Appl. Cryst. 2013. Vol. 46. P. 926-932. DOI: 10.1107/S0021889813012387

[11] de Boer D.K.G. // Phys. Rev. B. 1996. Vol. 53. P. 6048. DOI: 10.1103/PhysRevB.53.6048

[12] Ogilvy J.A. // Rep. Prog. Phys. 1987. Vol. 50. N 12. P. $1553-1608$.

[13] Kozhevnikov I.V., Pyatakhin M.V. // J. X-Ray Sci. Technol. 2000. Vol. 8. N 4. P. 253-275.

[14] Goray L.I. // J. Appl. Phys. 2010. Vol. 108. P. 033516. DOI:10.1063/1.3467937

[15] Svechnikov M. // J. Appl. Cryst. 2019. Vol. 53. N 1. P. 244-252.

[16] Goray L., Lubov M. // Opt. Express. 2015. Vol. 23. N 8. P. 10703-10713. DOI: 10.1364/OE.23.010703

[17] Lubov M., Goray L.J. // Synchrotron Rad. 2019. Vol. 26. P. 1539-1545. DOI: $10.1107 / \mathrm{S} 1600577519006337$

[18] Website of I. I. G., Inc.: [Интернет-ресурc] / URL: http://pcgrate.com (дата обращения 05.03.2020).

[19] Stearns D.G. // J. Appl. Phys. 1992. Vol. 71. N 9. P. 4286. DOI: $10.1063 / 1.350810$

[20] Stearns D.G., Gaines D.P., Sweeney D.W., Gullikson E.M. // J. Appl. Phys. 1998. Vol. 84. N 2. P. 1003-1028.

DOI: $10.1063 / 1.368098$

[21] Sinha S.K., Sirota E.B., Garoff S., Stanley H.B. // Phys. Rev. B. 1988. Vol. 38. P. 2297. DOI: 10.1103/PhysRevB.38.2297

[22] Center of X-ray Optics: [Интернет-ресурс] / URL: http://henke.lbl.gov/optical_constants/ (дата обращения 05.03.2020). 